\title{
Computational Method to Quantify the Evolution of Ocean Properties
}

\author{
O. Marcou \\ Université de Perpignan Via Domitia \\ Laboratoire IMAGES EA 4218 \\ 52 avenue Paul Alduy - 66860 Perpignan cedex, France
}

\author{
S. El Yacoubi \\ Université de Perpignan Via Domitia \\ Laboratoire IMAGES EA 4218 \\ 52 avenue Paul Alduy - 66860 Perpignan cedex, France
}

\author{
C. Goyet \\ Université de Perpignan Via Domitia \\ Laboratoire IMAGES EA 4218 \\ 52 avenue Paul Alduy - 66860 Perpignan cedex, France
}

\begin{abstract}
Understanding the recent trends in observable changes of the ocean physical and chemical properties is of great importance in order to foresee their future evolution and to quantify the probable contribution of anthropogenic pressure to these changes. For that matter, it is necessary to consider new methods and concepts for the analysis and modelling of ocean properties. In this paper, we introduce an original set of computational tools to process data measurements in order to study the evolution of some ocean properties. The method will be utilized to thoroughly analyze the evolution of an ocean property through a given period of time and in a whole water column by comparing its values at a given space/time coordinate to a well-defined reference value. Results will be exploited to emphasize the so-called spreadability phenomenon. Some insight will be given on the spreadability concept, based on systems theory. The ocean property that was chosen for the application presented in this paper was salinity, using data from the DYFAMED program for the period 1994-2010. Other analysis aspects could be studied using the proposed method.
\end{abstract}

\section{Keywords:}

Data Processing, Ocean Acidification, Spreadability, DYFAMED

\section{INTRODUCTION}

Since the industrial revolution, various physical and chemical changes have been observed in both the atmosphere and the oceans, including climate changes and ocean acidification. It is of course unclear to what extent these changes are correlated to mankind action. In the particular topic of ocean physical and chemical properties, a rather large amount of data has been obtained from various research campaigns. This paper will focus on a preliminary investigation of analysis and modeling methods and concepts, and on their application using data from the DYFAMED program (DYnamique des Flux Atmosphériques en MEDiterranée). For this study, the very basic ocean property of salinity will be considered, as a large set of data is available for it.
The measurements of basic physical ocean properties like temperature and salinity have been generally increasing during the second half of the 20th century in Western Mediterranean deep waters (WMDW). This increase has been recorded ever since the 1960s (see [6, 12, 9, 5, 1]).

Some data implies a sharp and accelerating increase during last years. For salinity in deep waters (around or below 2000m) in western Mediterranean Sea, Bethoux et al report an increase of about $1.1 \cdot 10^{-3} \mathrm{y}^{-1}$ since the 1960's [6, 4]. [15] reported an identical increase of $1.1 \cdot 10^{-3} \mathrm{y}^{-1}$ after 1960 and of $6 \cdot 10^{-4} \mathrm{y}^{-1}$ before 1960.

For the particular case of the DYFAMED site in the Ligurian see, several works also support this assertion. Notably, Marty et al [10] report an increase of $2.2 \cdot 10^{-3} \mathrm{y}^{-1}$ for the 1995-2005 period. Touratier et al in [14] estimate a similar value of $2 \cdot 10^{-3} \mathrm{y}^{-1}$ for the 1993-2005 period for the salinity increase at the DYFAMED site. Explanations about this evolution are highly debated, and some of them are related to anthropogenic pressure. Increases of salinity are linked to decreases in fresh water fluxes. This can be caused by a decrease of precipitations, or a reduction of rivers contributions, notably because of damming. The increase of WMDW salinity is probably the result of surface salinity increase in deep waters formation sites and of an increase of salinity in Levantine Intermediate Waters, usually formed in the eastern basin (see [13]). The characteristics of inflowing Atlantic waters may also be of some influence [11].

As was pointed out, mean values for salinity increase have been calculated by various authors over different time periods. These increases are usually calculated preferably in deep waters $(\approx 2000 \mathrm{~m})$, where long-term trends are more easily noticeable. Indeed, important seasonal and interannual variations exist in waters that are closer to the surface. However, it is still of interest to know the salinity trend in the whole water column. Such information is usually given in litterature in the form of contour plots of salinity itself (or whichever seawater property of interest). Here, an estimation of salinity increase relatively to a reference value will be considered, rather than salinity itself. 
Therefore, the aim of this paper is to propose a computational method for establishing a map of the increase of a given property compared to a pre-defined initial state. The notion of spreadability (a mathematical concept that originates in systems theory) will also be introduced in order to analyze the obtained results. These methods will then be applied to the salinity data available for the DYFAMED site.

\section{METHODS}

\subsection{Data}

In this study, hydrological data $(\mathrm{S}, \mathrm{T})$ will be used based on measurements made on the DYFAMED site (Fig. 1) located in the Ligurian Sea $\left(42^{\circ}=25 \mathrm{~N}, 7^{\circ} 59 \mathrm{E}\right)$, in the North-Western of the Mediterranean Sea, for the period 1994 to 2009. This data has a more or less monthly time periodicity (with some months missing). A given set of samples corresponds to a date at which a series of measurements were taken at several depths. The space periodicity of a series of samples varies : usually, one sample is taken every 2 meters, but it can also be 1 meter or 10 meters. Measurements also generally stop near the bottom (at more than $2000 \mathrm{~m}$ depth), but that maximum is also variable (it is sometimes reduced at $500 \mathrm{~m}$ or even 200m).

DYFAMED database (http://www.obs-vlfr.fr/dyfBase/) and all details on the collections methods are presented on the DYFAMED program homepage (http://www.obs-vlfr.fr/sodyf/home.htm/).

For simplicity, two coordinates will define the "position" of any existing sample : a time coordinate noted $t$ (expressed as a number of months) and a space coordinate within the water column $y$ (expressed as a pressure). A time-space grid is then defined with a fixed time step of 1 month and a fixed space step (which are usually choosen as 2 meters or 10 meters - converted in decibars since we actually use pressure and not depth).

Each actual sample may have either a pressure measurement, a depth measurement, or both of them. When the pressure measurement is available, it is used directly to determine the space coordinate of the sample. If there is no pressure measurement available, the depth measurement is then converted into a pressure.

Each sample is then affected to a given grid point of coordinates $(t, y)$ according to the following rules :

- Time : it must have been taken during the actual month corresponding to the $t$ value, with $t$ starting at 0 . For example, if the studied period begins at January 1st 1994, then a sample taken at January 2nd 1994 or at January 27th 1994 have a $t$ coordinate of 0 . A sample taken at date February 1 st 1994 would have a $t$ coordinate of 1 , and so on.

-Depth : its depth needs to be comprised within a given interval around the nominal depth of a given depth slice. The $0-2000 \mathrm{~m}$ depth interval that is studied is divided into slices with a resolution of either $2 \mathrm{~m}$ or $10 \mathrm{~m}$. If for example the chosen space resolution is $10 \mathrm{~m}$, then slices will be defined for at $10 \mathrm{~m}, 20 \mathrm{~m}$, $30 \mathrm{~m}$, etc. The authorized deviation around the nominal depth depends on the depth : it is usually chosen small at shallow depths, and larger at deeper depths (below 200m). Even though nominal depths are expressed in meters, the software actually work with decibars-converted values.

If a sample cannot be affected to any grid point because its depth is too far away from the nominal depth of any grid point, it is simply dropped.
The data is then reworked so that there is finally exactly one data point for each grid point (that is, each possible pair of month / depth slice). Each point can be either :

-One measurement (if one unique actual sample is available in the concerned month/depth slice).

- The average of several measurements (if several actual samples are available in a given month/depth slice).

- An interpolation computed from other points (if there is no sample at all in the considered month/depth slice).

The maximum depth that was considered in this paper is 2100 meters. The temporal limits of the studied dataset are January 1st 1994 and January 1st 2010.

\subsection{Interpolation method}

The method used to interpolate missing data was chosen so that it could be easily adapted to an irregular distribution of available data. Indeed, the available data utilized in this work do not correspond to a regular grid. Therefore, some of traditional methods for interpolation (like splines) cannot be used easily.

An inverse distance weighting interpolation method was chosen.

As previously defined, each "point" (either one measurement, average of a monthly set of measurements, or empty slot to be interpolated) possesses two coordinates : one time coordinate (noted $t$ ), expressed as the number of months since the beginning of the studied period, and one pressure coordinate (noted $y$ ), expressed in dbars.

First, the distance between two points $x_{1}$ and $x_{2}$ is defined the following way:

$$
d\left(x_{1}, x_{2}\right)=\sqrt{p_{y} \cdot\left(y\left(x_{1}\right)-y\left(x_{2}\right)\right)^{2}+p_{t} \cdot\left(t\left(x_{1}\right)-t\left(x_{2}\right)\right)^{2}}
$$

Where $y\left(x_{1}\right)$ and $y\left(x_{2}\right)$ are the space coordinates of respectively $x_{1}$ and $x_{2}$, and $t\left(x_{1}\right)$ and $t\left(x_{2}\right)$ are the time coordinates of respectively $x_{1}$ and $x_{2}$. Time and pressure are obviously not expressed in the same unit. Therefore, a specific weighting coefficient is affected to both of them in the distance formula. The weighting coefficient for the time coordinate is noted $p_{t}$ while the weighting coefficient for the pressure coordinate is noted $p_{y}$.

Interpolating the value of a given property in a given point consists in selecting a set of the nearest existing points (that is, all points located at a distance inferior to an arbitrarily fixed maximum distance noted $d_{\max }$ ), and then calculate a weighted average of the values of the property for each of the selected points.

The weighted average of an arbitrary property noted $u$ is calculated according to the following formula:

$$
u(x)=\sum_{i=1}^{n}\left(\frac{w_{i}(x) * u\left(x_{i}\right)}{\sum_{i=j}^{n}\left(w_{j}(x)\right)}\right)
$$

where $\left\{x_{1}, \ldots, x_{n}\right\}$ is the set of actual data points selected to interpolate the point $x$, with $w_{i}$ the weights associated to each selected point. These $w_{i}$ weights are computed as follows:

$$
w_{i}(x)=\frac{1}{d\left(x, x_{i}\right)^{p}}
$$

where $p$ is a parameter whose value is chosen arbitrarily. A high value for this parameter leads to give more importance to the nearest selected points. 

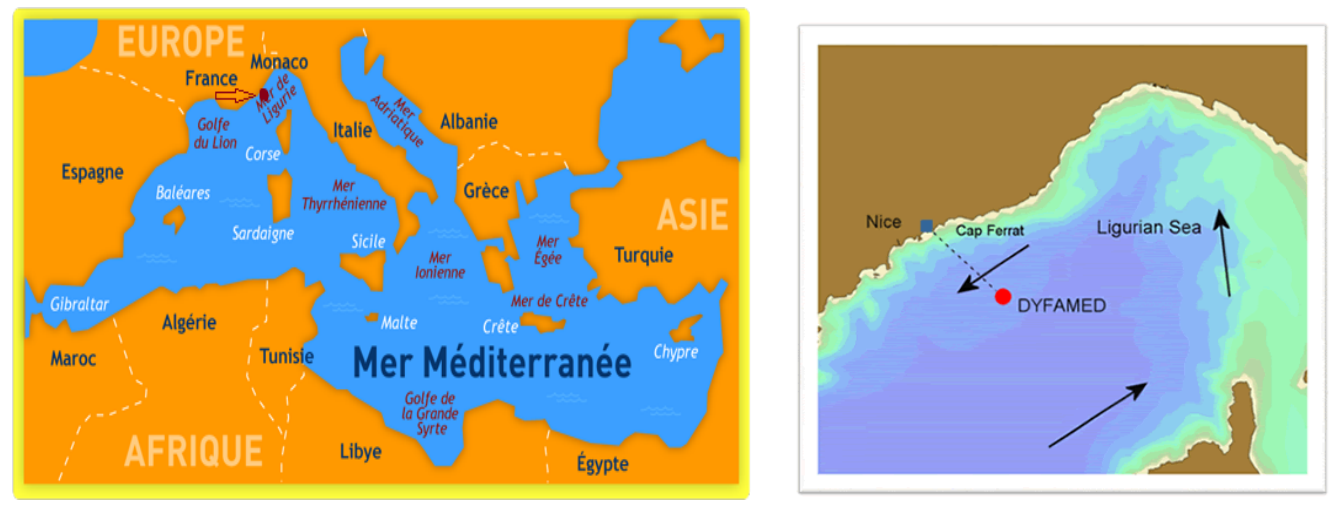

Fig. 1. Dyfamed location in the north of the Mediterranean Sea

In the present work, this method was used to interpolate salinity where no measurement was available.

\subsection{Implementation}

One of the important aspects of the present paper is that the processes described above have been computerized into an internally developed software that allows us to rapidly produce results without having to select and manipulate enormous amounts of data manually.

The first important feature of this software is the automatic importation, selection and sorting of the relevant data. The dataset used was the result of measurements series for the DYFAMED program amounting to a more or less 15 years period. Since for each year there is usually one dataset per month covering the whole water column (with one sample every 2 or 10 meters), the size of the total database is quite huge, and thus manual manipulation of this data to reduce it to the exact set of samples that is relevant for the considered application would be a challenge.

Therefore, we designed a data importation, selection and sorting utility. This tool imports a large number of text files containing different datasets, with possibly significant differences in the type or units of the properties recorded. During this operation, it operates a selection of the relevant data based on the considered properties or the studied time and space intervals, an elimination of incomplete samples, an automatic conversion of quantities expressed in a unit different than the one chosen and finally sorts the data according to relevant criteria.

After importation tools, various functionnalities have been added. Notably, an internal mathematical interpretor allows to calculate new fields based on the ones already existing. That mathematical interpretor currently comprises the possibility to calculate various basic statistics (notably averages, linear regressions...) as well as some functions that are more specific to our particular needs, like oceanography-centered calculation tools: for instance the resolution of all carbonate system properties from the values of two of them, unit conversion between pressure and depth, calculation of the Revelle factor, etc. The system also includes missing data interpolation tools based on the principles described in the previous section.

The final result of an automatic analysis by this software is a collection of text files containing the data and calculation results that were required. Density plot of particular fields can also be provided.

\subsection{Analysis methodology: spreadability concept}

The objective of the present work is to observe a potential overall tendancy for the evolution of salinity during the considered period. As it was previously mentioned, the considered "depth slices" represent usually $2 \mathrm{~m}$ or $10 \mathrm{~m}$. For each of these depths, one wishes to know how salinity evolved during the studied period, compared to its initial value. Therefore, it is first needed to determine an estimation of this "initial state", which will be called the salinity reference value.

A salinity reference value is calculated for each depth slice as the average value for salinity of the set of all points belonging to that depth slice, and whose date is inferior to a chosen maximum date. In other terms, a reference salinity average profile is defined and calculated on a given reference period. This reference period is included in the studied period, and it normally chosen at its beginning.

Therefore, in this work, salinity will not be studied directly, but rather the evolution of salinity with respect to its reference value. For each depth $y$ and time $t$ for which a measure of salinity is available and noted $\operatorname{Sal}(y, t)$, a new quantity $\operatorname{Sal}^{*}(y, t)$ is defined, which is calculated the following way:

$$
\operatorname{Sal}^{*}(y, t)=\operatorname{Sal}(y, t)-\operatorname{Sal}^{r e f}(y)
$$

Then, a new variable $\operatorname{Sal}^{b}(y, t)$ is defined again whose value depends on whether or not the salinity value at a given point is superior or inferior to the reference profile. The analysis will be performed through the prism of this new variable, which is defined as a function of salinity.

This variable is a boolean variable that is defined as follows:

$$
\begin{aligned}
& \operatorname{Sal}^{b}(y, t)=1 \text { if } \operatorname{Sal}^{*}(y, t)>0 \\
& \operatorname{Sal}^{b}(y, t)=0 \text { if } \operatorname{Sal}^{*}(y, t) \leq 0
\end{aligned}
$$

It can also be expressed in the following way :

$-S a l^{b}(y, t)$ is considered True at a given point $(y, t)$ if the salinity at this point is superior to the corresponding salinity reference value $\operatorname{Sal}^{r e f}(y)$,

$-\operatorname{Sal}^{b}(y, t)$ is considered False at a given point $(y, t)$ if the salinity at this point is inferior to the corresponding salinity reference value $\operatorname{Sal}^{\text {ref }}(y)$. 
In the results that will be showed, the reference period was chosen between 1994/01/01 (beginning of the overall study period) and 1996/01/01.

The previously defined variables will be analyzed in relation with the concept of spreadability which describes the phenomenon of spatial expansion with time of a given "property" [7, 8]. By "property", here we do not mean a physical or chemical property in the common sense as used in oceanography works, but a given condition that may or may not be satisfied in any point of the considered spatial domain at a given time. Spreadable phenomena can be observed in various environmental processes, as pollution, vegetation cover, population infection, presence of a particular biological species, etc. The primary idea behind the spreadability concept is that subdomains where a given spatial property is verified are nondecreasing.

Let $P$ be a spatial property which aims to be spread or resorbed over time. Let $\Omega$ be a one-dimensional spatial domain constituted of cells $c$, where each cell is characterized by a state $s_{t}(c)$ that varies with time $t$. We denote by $S$ the system whose state $S_{t}$ at time $t$ is constituted by the set of all cell states $s_{t}(c)$. The system state $S_{t}$ can be given according to a defined dynamics or, as it will be in this work, defined by direct measurements. The sequence of subdomains $\left\{\omega_{t}\right\}_{t \geq 0}$ where the property $P$ is satisfied at time $t$ is given by :

$$
\omega_{t}=\left\{c \in \Omega \mid P s_{t}(c)\right\}
$$

$P$ is defined as follows :

$$
\forall c \in \Omega, P s_{t}(c) \Leftrightarrow \operatorname{Sal}(y, t)>\operatorname{Sal}^{r e f}(y)
$$

where $y$ is the spatial coordinate of cell $c$.

Then, a definition for the spreadability concept is needed.

Definition 1 The system $S$ is said to be $P$-spreadable ( $P$ resorbable) if the sequence $\left\{\omega_{t}\right\}_{t \geq 0}$ is increasing (decreasing), that is :

$$
\omega_{t} \subseteq \omega_{t+1} \quad\left(\omega_{t} \supseteq \omega_{t+1}\right) \quad \forall t \geq 0
$$

Thus, the property $P$ will be considered verified when $\operatorname{Sal}^{b}(y, t)$ is true. However, at this point, it can already be objected that the previous definition for spreadability will not be very helpful. Indeed, it is required that at each time $t$ the subset $\omega_{t}$ (comprising all the cells verifying the property) contains at least exactly the totality of the subset at time $t-1, \omega_{t-1}$, which means each cell verifying the property will then verify it forever. Obviously, in an actual ocean water column this condition will rarely be satisfied.

Therefore, another variant of the spreadability concept will rather be used [3, 2] : $\mu$-spreadability (or extended spreadability, or spreadability in the sense of measures). In this variant, the areas of subsets $\omega_{t}$ are what is supposed to be increasing.

Definition 2 The system $S$ is said to be $P$-spreadable ( $P$ resorbable) in the sense of areas or $\mu$-spreadable ( $\mu$-resorbable) during a time interval $I=\left[t_{0}, T\right]$ if for any $t, s$ with $t_{0} \leq t \leq$ $s \leq T:$

$$
\operatorname{mes}\left(\omega_{t} \backslash \omega_{s}\right) \leq \operatorname{mes}\left(\omega_{s} \backslash \omega_{t}\right) \quad\left(\operatorname{mes}\left(\omega_{t} \backslash \omega_{s}\right) \geq \operatorname{mes}\left(\omega_{s} \backslash \omega_{t}\right)\right)
$$

This definition (for $\mu$-spreadability) means that the surface gain (areas where the property spread) during the time interval $[t, s]$ is greater than the surface loss (areas where the property resorbed) during that interval (of course, it is exactly the contrary for $\mu$ resorbability).

\section{RESULTS}

An analysis of salinity during the 1994-2009 period and the 0$2100 \mathrm{~m}$ depth interval was performed according to the previously defined methodology. Results are shown for four different depth intervals: the interval 0-200m (Surface Water or Modified Atlantic Waters - MAW), the interval 200-600m (Levantin Intermediate Waters - LIW), the interval 600-1600m (upper Western Mediterranean Deep Waters - WMDW) and the interval 1600-2100m (pure WMDW). These water masses and the respective depths they approximately recover at the DYFAMED site are common knowledge (see for instance [10]).

The salinity reference profile is shown in Fig. 2 a colormap showing the boolean variable $\mathrm{Sal}^{b}$ (Fig. 3- which means showing the points where salinity is above the reference value), a colormap showing the continuous variable $\mathrm{Sal}^{*}$ (Fig. 4- salinity minus reference salinity), a colormap showing the places where the salinity needed to be interpolated (Fig. 5), and a figure showing respectively for each year and for each month (Fig. 6 and 7) the proportion of points where salinity was above the reference value. This last figure makes use of the $\mu$-spreadability concept that was presented in Def. $2:$ in this work, the space domain $\Omega$ is the aforementioned water column, the cells $c$ are the given individual depths at which measurements (or interpolations) are available, the cell states $s_{t}(c)$ are the salinity values, and the property will be the fact for the salinity value to be above the corresponding reference Salinity (also, one can consider the state to be the value of the boolean variable $S a l^{b}$ and the property to be verified when $S a l^{b}$ is true). Therefore, the left part of Fig. 6 and 7 is simply the measure of $\omega_{t}$, the subdomain of $\omega$ into which the property is verified.

A first and preliminary remark at this points is that in Fig. 5 some areas can be clearly observed where the quantity of missing data is high enough to make us careful about interpretations of the results in these specific areas. The main areas concerned are those located at the extremities of the studied period. In order to have reliable interpolations, it is obvious that actual data should be present before and after the interpolated points. This is not the case in some areas, notably in upper and pure WMDW close to the beginning or the end of the studied period.

In the rest of the text, the term "high salinity areas" will designate the areas where salinity is greater than the reference value, and the term "low salinity areas" will designate the areas where salinity is lower then the reference value.

Examining the overall tendancy in colormaps for depths $0-2100 \mathrm{~m}$ (Fig 3), it appears that high and low salinity areas seem rather well balanced in shallow depths, while in deeper depths there is a prominence of high salinity areas, except at the beginning of the studied period where the two types of areas are also well balanced. However, since it corresponds to the reference period, it is logical that high and low salinity areas are balanced in that domain.

A more in-depth analysis can be done by analyzing separately the different depth domains $(0-200 \mathrm{~m}, 200-600 \mathrm{~m}, 600-1600 \mathrm{~m}, 1600-$ $2100 \mathrm{~m}$ ) and by summarizing the proportion of points having high salinity depending on time in each of these domains. The latter is done in Fig. 7

It is confirmed that in shallow depths $(0-200 \mathrm{~m})$ the areas of high and low salinities are effectively well-balanced (Fig 3 and 7 ) throughout the studied time period.

The same observation can roughly be made concerning the intermediate depths $(200-600 \mathrm{~m})$, but it is mostly valid for depths ranging from $200 \mathrm{~m}$ to approximately $400 \mathrm{~m}$, during years 1997 and 2005 . For 400-600m, there are mostly high salinity areas between 1997 


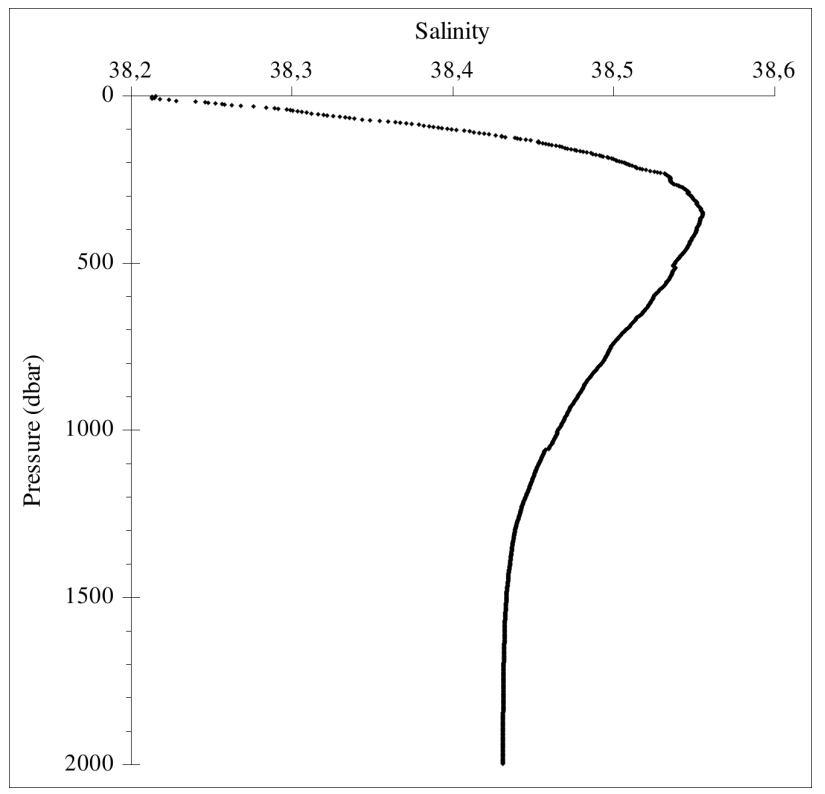

Fig. 2. An example of Salinity reference profile.

and 2005. A particular phenomena happens in 2005/2006 where low salinity areas suddenly appear.

On the other hand, when considering the depth slices $600-1600 \mathrm{~m}$, one can clearly see an important difference between before and after a date roughly around 1997, in Fig $3 \mathrm{c}$ and $3 \mathrm{~d}$ as well as in Fig 7/e to 7 h. Until 1997 (approximately), areas of low and high salinity are more or less equally present, while after 1997 areas of high salinity are the majority by a large margin.

In the 1600-2100m domain, high and low salinity areas are equally distributed until almost 1998. After, there are almost only high salinity areas. Looking to Fig. 4d, one can also state that while $S a l^{*}$ seems to remain more or less constant (with maybe a slight tendancy to increase) during years 1998 to 2006, a sudden increase is observable in year 2006. Afterwards, $S a l^{*}$ remains stable for all following years.

One can also notably see that this year 2006 also shows remarkable changes for other depth domains. One can observe a phenomenon of decreasing salinity between years 2005 and 2008 and between approximately 200 and $900 \mathrm{~m}$, that is visible in Figs. 3 b, 3 c, 4 b and $4 \mathrm{c}$. This spectacular decrease diminishes then disappears between years 2007 and 2009 .

\section{DISCUSSION}

The particularity of this study is that the presented results focus specifically on showing the evolution of the chosen ocean property compared to its level in the period 1994-1996.

Observations concerning the evolution of salinity in the DYFAMED water column have already been made by other authors in previous publications. The results shown in the present work will notably be compared with results from [10], where the evolution of salinity is studied between 1994 and 2006.

The data in the WMDW showed, according to [10], a slight but regular increase of salinity from 1995 onwards, until an abrupt increase in 2006. One can find in Fig. 4d that it is globally verified : as was already stated, what one observes in this figure can be described as an either constant or slight increasing salinity before
2006 (with alternations of low and high salinities before 1998), and in 2006 a brutal increase is clearly visible. It should also be noted that this increase is still persisting in years 2007, 2008 and 2009 that were not available in Marty et al's work.

In the upper WMDW (600-1600m), Marty and Chiavérini also report a slight increase until year 2006. However, they observe a decrease of salinity in the intermediate deep layer, in opposite to what happens in the pure WMDW. One can see in Fig. 4 c that this is explainable by a strong salinity decrease also affecting the LIW, occurring between 200 and $900 \mathrm{~m}$ approximately. However, it seems that starting 2009, high salinities appear again.

Concerning intermediate waters (LIW), Marty and Chiavérini observe that the salinity is relatively constant or slightly increasing from 1995 to 2004, and then decreases strongly during years 2005 and 2006. This brutal decrease can be observed in Fig. 4 b.

In surface waters (MAW, $0-200 \mathrm{~m}$ ), the main observable phenomena are usually strong seasonal variations. Marty and Chiavérini report these seasonal variations as well as a global trend to stability for salinity, until years 2002/2003 onwards when a regular increase is observed. One also observes in Fig. 4 a very noisy but overall stable values until approximately 2004 (with seasonal alternations of low and high salinities). There are then several years of high salinity between approximately 2004 and 2008. Starting 2008, there is again an alternation of low and high salinity periods, but with globally fewer low salinity periods.

Another important observation of Marty and Chiavérini in [10] is the phenomenon of almost complete homogenization of the water column that happened in 2006. In [10], this phenomenon is best observed in contour plots. However, it is difficult to identify in a direct manner this phenomenon in the figures presented in the present work, due to the fact that what is captured is salinity minus a mean salinity, and not salinity directly. The most easily observable consequence of the water column homogenization in Figs. 4 a to 4 d is probably the brutal and important decrease of $S a l^{*}$ that one observes in Figs $4 \mathrm{~b}$ and $4 \mathrm{c}$. Indeed, homogenization of the water column means that salinity will become higher at depths where it is usually low and lower in depths where it is usually high. Typical salinity profiles (such as the ones in Fig. 2) show that salinity presents a maximum between 200 and $800 \mathrm{~m}$ approximately, which correspond well to the region in which one observes the decrease of $S a l^{*}$. Incidentally, one can also see an increase of $S a l^{*}$ in WMDW, where salinity is low.

The most important global observation that can be noted is that the salinity clearly exhibits a strong tendancy to become superior to its levels of the reference period 1994-1996. This is most noticeable in the $1600-2100 \mathrm{~m}$ area (Figs. $3 \mathrm{~d}$ and Figs. 4 d) where the longterm trends can be best observed as well as in the $600 \mathrm{~m}-1600 \mathrm{~m}$ area (Figs. $4 \mathrm{c}$ and $4 \mathrm{~d}$ ).

A noticeable exception to that general rule is the event of water column homogenization that happened in 2006, which leads to a decrease of salinity in the $200-800 \mathrm{~m}$ depths range.

This tendancy is less clear in more shallow depths as was noted by the analysis of the MAW and LIW regions (0-600m depth domains). The salinity in this area is extremely random since it is strongly affected by all surface exchanges, and therefore it is difficult to observe an overall tendancy to salinity increase, at least for the 1994-2004 period. Such a tendancy to increase seems noticeable in the 2004-2008 period, but it is likely partly a manifestation of the 2006 homogenization event.

In order to analyze these results from a spreadability perspective, consider the graphics shown in Fig. 7 and 6 will now be considered. As it was already precised, the Fig. 6 show the evolution of the size of the subdomain $\omega_{t}$ of the water column that verify the property 


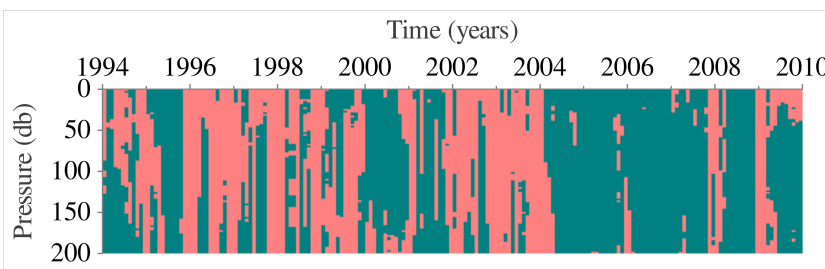

(a) $0-200 \mathrm{~m}$.

Time (years)

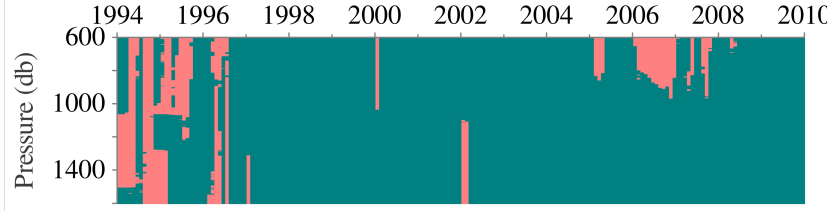

(c) $600-1600 \mathrm{~m}$.

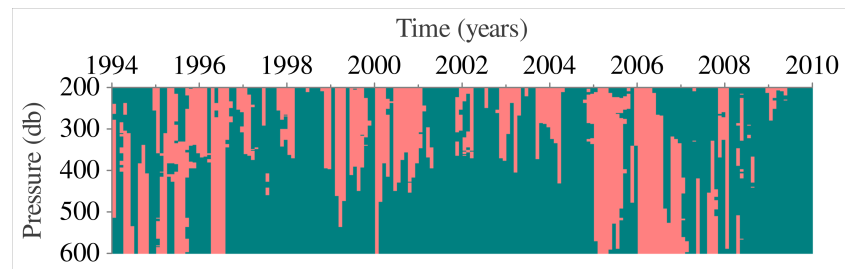

(b) $200-600 \mathrm{~m}$.

Time (years)

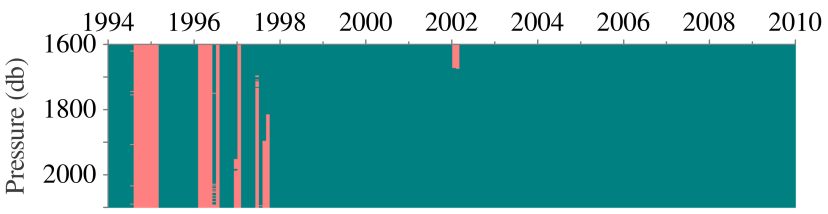

(d) $1600-2100 \mathrm{~m}$.

Fig. 3. Evolution of the variable $S a l^{b}$ in function of time and space. Points where salinity is greater than the reference value are blue-colored, while points where salinity is lower then the reference value are red-colored.

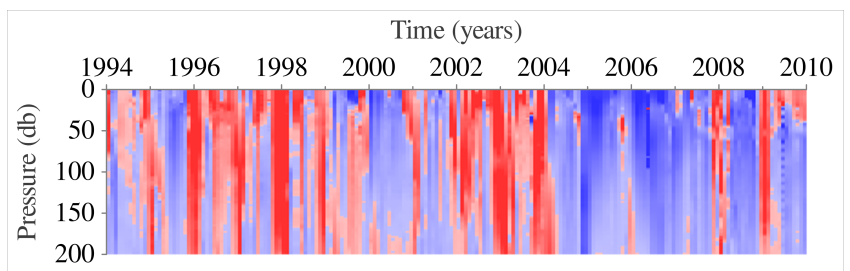

(a) $0-200 \mathrm{~m}$.

Time (years)

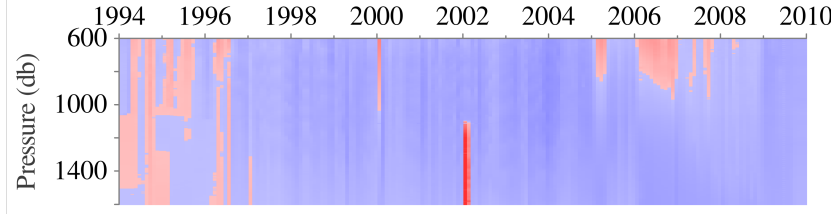

(c) $600-1600 \mathrm{~m}$.

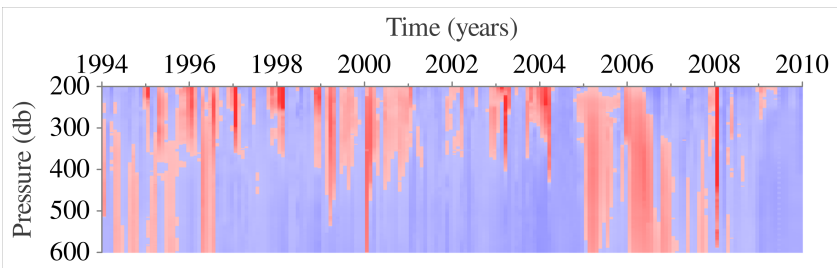

(b) $200-600 \mathrm{~m}$.

Time (years)

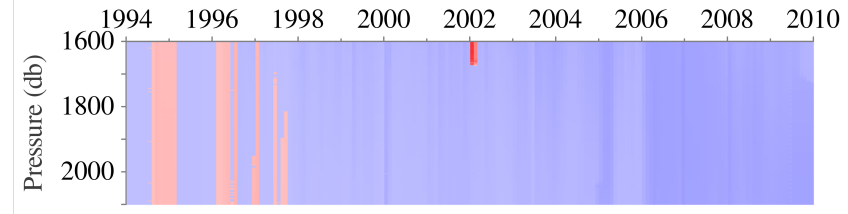

(d) $1600-2100 \mathrm{~m}$.

Fig. 4. Evolution of the variable $S a l^{*}$ as a function of time and space. Points where salinity is greater than the reference value are blue-colored, while points where salinity is lower then the reference value are red-colored.

$\operatorname{Sal}(y, t)>\operatorname{Sal}^{\text {ref }}(y)$ during the entire studied period. Fig. 7 does the same for the different water masses. This evolution is showed for monthly (left half of Fig. 7 and 6) as well as yearly (right half of Fig. 7] and 6 timescales.

At first glance, the monthly data (figures $7 \mathrm{a}, 7 \mathrm{c}, 7 \mathrm{e}, 7 \mathrm{~g}$ ) are generally very noisy. However, they still exhibit a clear tendancy to increase, at least for waters deeper than $600 \mathrm{~m}$ (Fig. $7 \mathrm{le}$ and $7 \mathrm{lg}$ ). When one considers the yearly data (right half of Fig. 7 and 6 , however, one can see general tendancies. In Fig. 6 (for the whole water column, $0-2100 \mathrm{~m}$ ), a clear succession of spread and resorbtion periods can be identified between 1994 and 2004, with peaks in 1995, 1998, 2001 and 2004, which means it seems to present a 3-years periodicity. This apparent periodicity seems to disappear after 2004. For surface waters and LIW, data seems noisy and quite difficult to interpret. However, the situation is different in deep waters. The upper WMDW also exhibits a succession of spread and resorbtion periods : at the beginning, the proportion of sites verify- ing $\operatorname{Sal}(y, t)>\operatorname{Sal}^{r e f}(y)$ increases until it reaches $100 \%$. Then it stays at $100 \%$ with occurrences of sudden decreases that reverse rapidly. In pure WMDW, one observes a very quick alternation of spread/resorbtion for the first four years, and then the proportion of sites verifying $\operatorname{Sal}(y, t)>\operatorname{Sal}^{\text {ref }}(y)$ stays almost constantly at its maximum. The concept of $\mu$-spreadability thus seems applicable to annual data of the high salinity cells proportion in deep waters.

\section{CONCLUSION}

This paper presents a method consisting in analyzing an ocean property not directly, but by first calculating the difference between the said property and a reference value chosen to be as close as possible to the supposed value at the beginning of the studied period. Therefore, the evolution of the property compared to the initial state can be visualized in a more direct manner. This method has been 


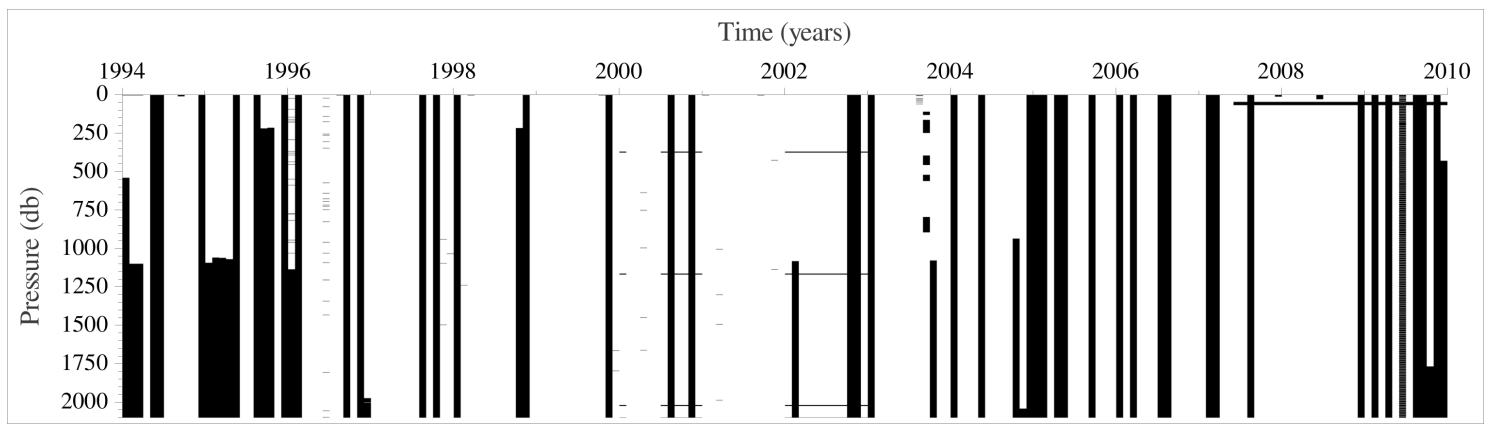

Fig. 5. Data provided by interpolation (in gray).

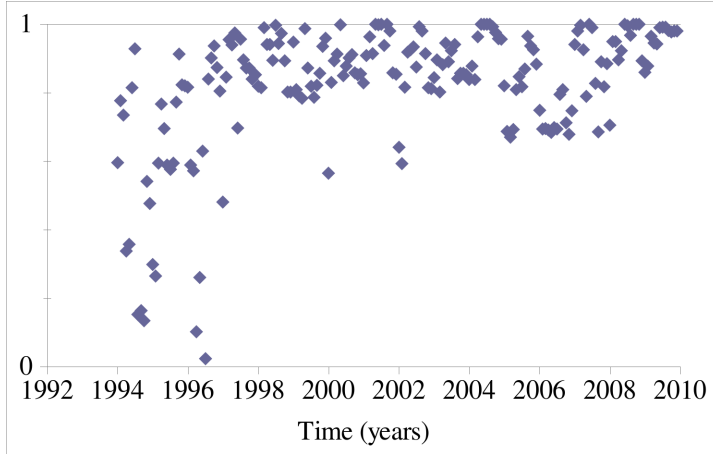

(a) 0-2100m, monthly.

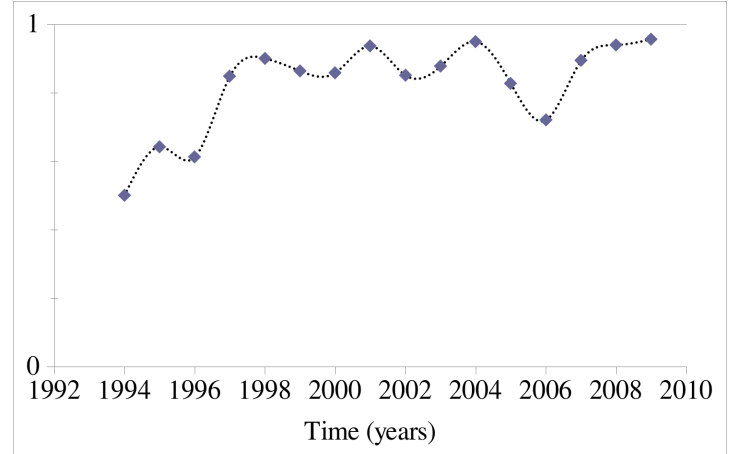

(b) $0-2100 \mathrm{~m}$, annual.

Fig. 6. Left-hand side : for depth domain $0-2100 \mathrm{~m}$, evolution of the proportion of monthly measurements verifying the property $S a l(y, t)>S a l^{\text {ref }}(y)$ (i.e., measure of the subdomain $\omega_{t}$ of the water column $\Omega$ where the property is verified) for the period 1994-2009. Right-hand side : yearly proportion of measurements verifying the property.

tested for salinity at the DYFAMED site in western mediterranean for the period 1994-2009.

One can clearly observe that salinity in WMDW after the reference period is at higher levels than what it was during the reference period. This is especially visible in pure WMDW (deeper than $1600 \mathrm{~m}$ ), but also in the upper WMDW (deeper than $600 \mathrm{~m}$ ) with some notable exceptions. The most noticeable exception is in the upper WMWD, close to the LIW waters, where the water column homogenization leads to a decrease of salinity in a certain depth domain in 2006 and later on. This water column homogenization is incidentally less easily identifiable than simply looking into salinity itself, since it is manifested by an apparent increase of salinity in areas where salinity is usually low and an apparent decrease of salinity in areas where salinity is usually high. Globally, in deep waters the proportion of high salinity cells increases quickly with time until it reaches its maximum, after which it resorbates and expands again several times, with the 2006 event being the most noticeable.

It can also be noticed that observations one can make when studying $S a l-S a l_{r e f}$ confirm observations from authors who previously studied the same dataset, which is a requirement to confirm the method's reliability. More specifically, the method allows to observe in a direct way the periods and areas where salinity was higher than its reference value (notably in Figs. 4 and 3 ) as well as to estimate the proportion of the water column verifying that property (see Fig. 7). At the end of the studied period (that is, ap- proximately two years more than the period studied by Marty and Chiavérini), it is also shown that the situation seems to return to a state without water column homogenization. However, this is not exactly the same situation that existed before the 2006 event : the overall salinity seems to have increased.

Potential perspectives of this work include applications to other properties, as well as refining the definition of the reference values that was used to estimate the property increase. It is notably clear that the reference period would be best estimated on a longer time period, and on a wider perspective repeated measurements over several decades would be necessary to fully quantify the evolution of seawater properties. Provided that a sufficient quantity of data is available, this procedure could easily be adapted to other ocean properties whose variations due to climate change and anthropogenic $\mathrm{CO}_{2}$ increase have a direct and significant impact on oceans chemical and biological equilibria (like $\mathrm{pH}$ or $\mathrm{CO}_{2}$ ). In this case, the reference profile for the studied ocean property could be chosen by considering, for example, criteria like risk thresholds. This method could then become a tool for risk assessment.

\section{Acknowledgments}

This is a contribution of the European project FP7 "Mediterranean Sea Acidification in a changing climate" project (MedSea; grant agreement 265103). 


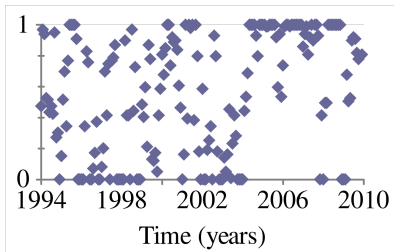

(a) $0-200 \mathrm{~m}$, monthly.

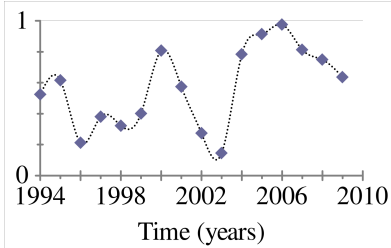

(b) 0-200m, annual.

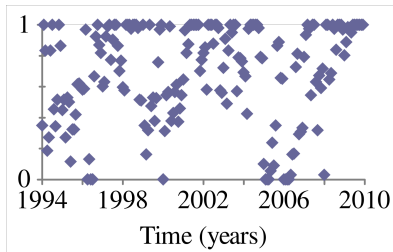

(c) $200-600 \mathrm{~m}$, monthly.

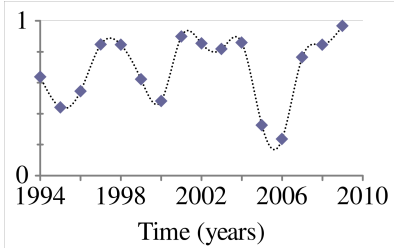

(d) 200-600m, annual.

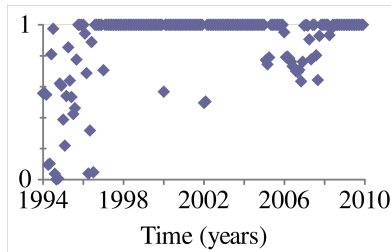

(e) $600-1600 \mathrm{~m}$, monthly.

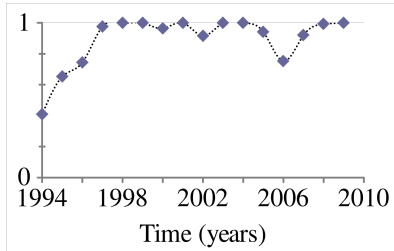

(f) $600-1600 \mathrm{~m}$, annual

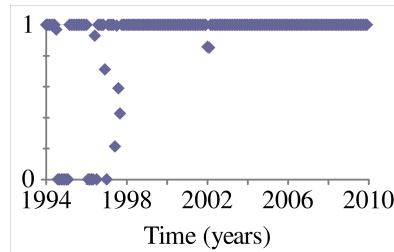

(g) 1600-2100m, monthly.

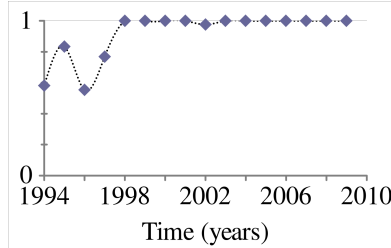

(h) $1600-2100 \mathrm{~m}$, annual.

Fig. 7. Left-hand side : for depth domains 0-200m, 200-600m, 600-1600m, 1600-2100m, evolution of the proportion of monthly measurements verifying the property $\operatorname{Sal}(y, t)>\operatorname{Sal}{ }^{\text {ref }}(y)$ (i.e., measure of the subdomain $\omega_{t}$ of the water column $\Omega$ where the property is verified) for the period $1994-2009$. Right-hand side : yearly proportion of measurements verifying the property.

\section{REFERENCES}

[1] M. Astraldi, G. P. Gasparini, A. Vetrano, and S. Vignudelli. Hydrographic characteristics and internal variability of water masses in the central mediterranean. Deep-Sea Res. Pt. I, 49:661-680, 2002.

[2] A. Bernoussi, A. El-Jai, and A.J. Pritchard. Spreadability and evolving interfaces. Int. J. System Science, 32:1217-1232, 2001.

[3] A. Bernoussi and A. El Jai. New approach of spreadability. Math. Comput. Model., 31:93-109, 2000.

[4] J. P. Bethoux, B. Gentili, , and D. Tailliez. Warming and freshwater budget change in the mediterranean since the 1940s, their possible relation to the greenhouse effect. Geophys. Res. Lett., 25:1023-1026, 1998.

[5] J. P. Bethoux and B. Gentili. Functioning of the mediterranean sea: past and present changes related to freshwater input and climate change. J. Marine Syst., 20:33-47, 1999.

[6] J. P. Bethoux, B. Gentili, J. Raunet, and D. Tailliez. Warming trend in the western mediterranean deep water. Nature, 357:660-662, 1990.

[7] A. El-Jai and K. Kassara. Spreadable distributed systems. Math. Comput. Model., 20:47-64, 1994.

[8] S. El-Yacoubi and A. El-Jai. Cellular automata and spreadability. Int. J. Math. Comp. Mod.., 36:1059-1074, 2002.

[9] G. K. Krahmann and F. Schott. Long term increases in western mediterranean salinities and temperature: anthropogenic and climatic sources. Geophys. Res. Lett., 25:4209-4212, 1998.

[10] J.C. Marty and J. Chiavérini. Hydrological changes in the ligurian sea (nw mediterranean, dyfamed site) during 1995-2007 and biogeochemical consequences. Biogeosciences Discuss., $7: 1377-1406,2010$

[11] C. Millot, J. Candela, J. L. Fuda, and Y. Tber. Large warming and salinification of the mediterranean outflow due to changes in its composition. Deep-Sea Res. Pt. I, 53:656-666, 2006.
[12] E. J. Rohling and H. L. Bryden. Man-induced salinity and temperature increases in the western mediterranean deep water. J. Geophys. Res., 97:11191-11198, 1992.

[13] N. Skliris, S. Sopfianos, and A. Lascaratos. Hydrological changes in the mediterranean sea in relation to changes in the freshwater budget: a numerical modelling study. J. Marine Syst., 65:400-416, 2007.

[14] F. Touratier and C. Goyet. Decadal evolution of anthropogenic co2 in the northwestern mediterranean sea from the mid-1990s to the mid-2000s. Deep Sea Research, 56:17081716, 2009.

[15] M.N. Tsimplis and T.F. Baker. Sea level drop in the mediterranean sea: An indicator of deep water salinity and temperature changes? Geophys. Res. Let., 27(12):1731-1734, 2000. 